\title{
Survey: IoT Vehicular Information Network
}

\author{
Prarna Dhar ${ }^{1}$, Poonam Gupta ${ }^{2}$ \\ ${ }^{1}$ Pune University, Computer Department, Pune, India \\ ${ }^{2}$ Professor, Pune University, Computer Department, Pune, India
}

\begin{abstract}
Inter vehicular communication is a technology where vehicles act as different nodes to form a network. In a vehicular network different vehicles communicate among each other via wireless access.Authentication is very crucial security service for inter vehicular communication (IVC) in Vehicular Information Network. It is because, protecting vehicles from any attempt to cause damage (misuse) to their private data and the attacks on their privacy. In this survey paper, we investigate the authentication issues for vehicular information network architecture based on the communication principle of named data networking (NDN). This paper surveys the most emerging paradigm of NDN in vehicular information network. So, we aims this survey paper helps to improve content naming, addressing, data aggregation and mobility for IVC in the vehicular information network.
\end{abstract}

Keywords: Internet of Things (IoT), named data networking, inter vehicular communication (IVC), Vehicular information network, MQTT

\section{Introduction}

Internet of Things(IoT) and vehicular information network technologies are one of the emerging technologies worldwide. Now a days vehicular information network has its great significance in IoT areas. In vehicular information network technology, vehicles act as nodes in a network to form mobile network so that different nodes(vehicles) can communicate with each other in a network. Nodes carry out communication with each other by some wireless access or by aggregator [1]. For communication purpose nodes or vehicles should be in range of 300 meters approximately so that nodes can communicate with other nodes or routes easily. Vehicles can retrieve more information (e.g. traffic information, road, and weather conditions) while on the road. Moreover, vehicles have capability to sense objects around them and provide information to end user. Vast amount of data processing and acquisition by inter vehicle communication (IVC) are done via intelligent vehicularcomputing platforms. Drivers can easily receive or send realtime information with less delay and even other transportation authorities can acquire real-time information about traffic on road and also about those vehicles who violate the traffic laws. In vehicular information network different number of services can be supported through inter vehicular communication and this is known for intelligent transportation system(ITS). There are different services that are supported through IVC are meant to be like exchange information of road safety, smart traffic management, information about navigation, information smart parking, alert information to drivers about road congestion, accidents, weather conditions, offering information for tourists, targeted advertisement for local services. Thus we need an efficient and scalable network architecture which support all the mentioned services or applications and vast amount of information that is to be expected to exchange in the vehicular information network.

In vehicular information network it is to be expected that there are two types of applications utilized for a broad range. One is safety applications and other is non-safety applications. Safety applications are those which provide some security to applications and non-safety applications are those which provide information about collision warnings, road navigation, traffic information and mobility information. In vehicular information networks, the user authentication is a crucial security service for access control in both intervehicle and vehicle-wireless access communication [7]. On the other hand, protection of vehicles from the misuse of their private data by someone else and the attacks on their privacy, so that one be capable to investigate the accidents or attacks for non-repudiation. Peculiarly, safety applications require a strong mutual authentication, because most of the safetyrelated messages may contain life-critical information.In the vehicular information network there are many routing protocols and identification schemes, if they still follow the traditional TCP/IP protocol then the various issues related to the security, scalability and efficiency of the current internet architecture are all inherited. Now a days, it has been researched that over the internet content or data has become the heart of all communications. Current internet architecture is not so efficient and scalable. It has few flaws, so the concept of information -centric networking was proposed to reached out those flaws. ICN introduces named data as a core internet principle so that it provides in-netwrok caching, named-based routing and scalable,cost-efficient and security.

\section{Literature Survey}

\subsection{Overview of ICN}

The concept of current architecture of internet is totally based on a host-centric communication so that one host(node) can communicate with end host. The internet usage involves the million number of users interest of accessing huge amount of information from internet with their respective physical locations. By emerging huge interest of users leads to the paradigm shift of the current architecture of internet which offers better security and mobility management which brings radical change in the current architecture of internet. 


\section{International Journal of Science and Research (IJSR) \\ ISSN (Online): 2319-7064}

Index Copernicus Value (2013): 6.14 $\mid$ Impact Factor (2014): 5.611

The internet and new applications are emerging tremendously just to fulfill all the emerging needs of users that are connected to internet worldwide. The newly emerging needs are to support scalable distribution of content(data), the mobility management, the security issues and so on. Therefore, researchers put great effort to initiate the topic of internet's architecture of Clean Slate Design. So the concept of ICN(Information -Centric networking) with different solutions is proposed [5].This concept aims to redesign the current internet architecture with the prespective of information -centric networking. There are number of recent research projects which adopt the idea of redesigning the current architecture of internet these are Data-Oriented Network Architecture (DONA), Publish-Subscribe Internet Technology, Named Data Networking (NDN) and Network of Information. All these projects aims the solutions of content-naming techniques and routing schemes that will be efficient and scalable for current Internet architecture. For better solution NDN performs best content routing on the basis of content name.NDN does not pay any attention on the location information like IP address which are used in the current internet architecture TCP/IP. NDN consist hierarchical content naming scheme and packet routing is on the basis of name-routing [1]. This newly developing architecture of internet is proving better suited for accessing data efficiently and is scalable in distributing large-scale content.

\subsection{Vehicular Information Network}

There are millions of vehicles that are connected with internet and also with each other. There are several standards who have given much attention for the development of vehicular information network in reality. The main aim of vehicular networks is to improve safety and efficiency in transportation. Vehicular networking shows its great importance in the deployment of vehicle-to-infrastructure network and also Vehicle-to-Vehicle communication network $[2]$.

Now a days vehicular information network forms humancentric ecosystem which helps in application sensing is growing so rapidly for data collection. For sensing platform there are number of devices like GPS device,RFID devices used for sensing the object to collect the data. Thus the collected data in vehicular information network is done by GPS which helps in monitoring the traffic, coordinate values of latitude and longitude,also traces pollution content in air and also give alert to drivers about road condition. Considering all these points there should be the architecture of vehicular information network which support or cover the mobility management of vehicles but for that GPS should be installed in vehicles which helps in getting current location of any object. Fig.1 illustrates how vehicular information network plays role when number of vehicles are connected with each other in a network.

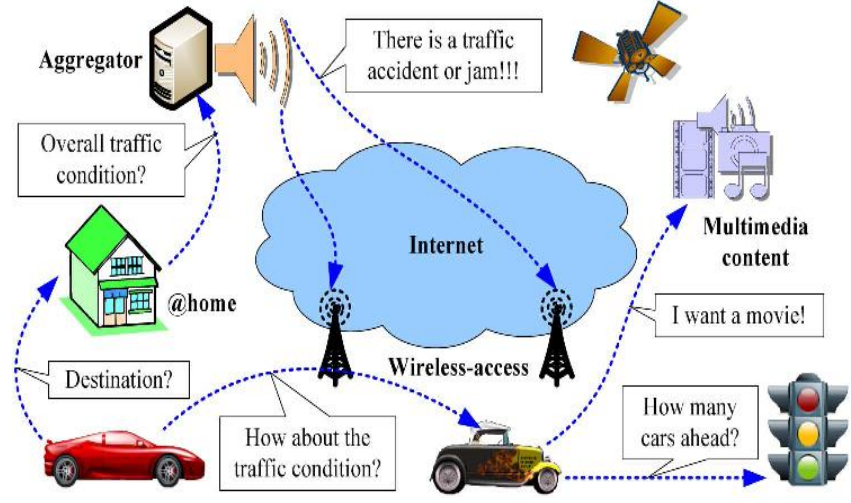

Figure 1: Vehicular information network

The above fig.1. clearly depicts about how vehicles communicate with each other in a network. Following are the communication cases.

1) Vehicles can sense the information of surrounding objects through inbuilt sensors and then disseminate the data by using pull based or push based communication technique through the network.

2)In an ad hoc way, vehicles can communicate with other vehicles to send or receive the data related to traffic information, road information and other data.

3)In fig.1 there is an aggregator which acts as a server. Aggregator is used for collection and processing of data. Aggregator also send the processed data on demand to other vehicles.

Based on all above communication cases, the main focus of this survey is to develop such a network which supports ad hoc network, mobility management and different schemes of communication for different applications in vehicular information network [5].

\subsection{NDN in Vehicular Information Network}

The architecture of a Named data networking is proposed by Jacobson is totally based on the concept of content- centric networking. In NDN communication is initiated by the consumer [1],[2]. Consumer sends the signaling message which is called as an Interest packet. Interest packets are those packets which indicates the desired content of consumer. When consumer sends the interest packet, there is a router who receives those interest packet by consumers. Router searches the data content in cache store(CS). If the router found the data copy in its cache, then it sends back replay as a data packet to the consumer. If the router does not found the requested content in its cache then it searches in the next-hop address in Forwarding information base(FIB). In forwarding information base it checks the longest prefix match of the desired data in its table [3]. If its match doesn't occur then router store that information in its PIT that is pending interest table. Fig.2. shows the packet routing. 


\section{International Journal of Science and Research (IJSR) \\ ISSN (Online): 2319-7064}

Index Copernicus Value (2013): 6.14 | Impact Factor (2014): 5.611

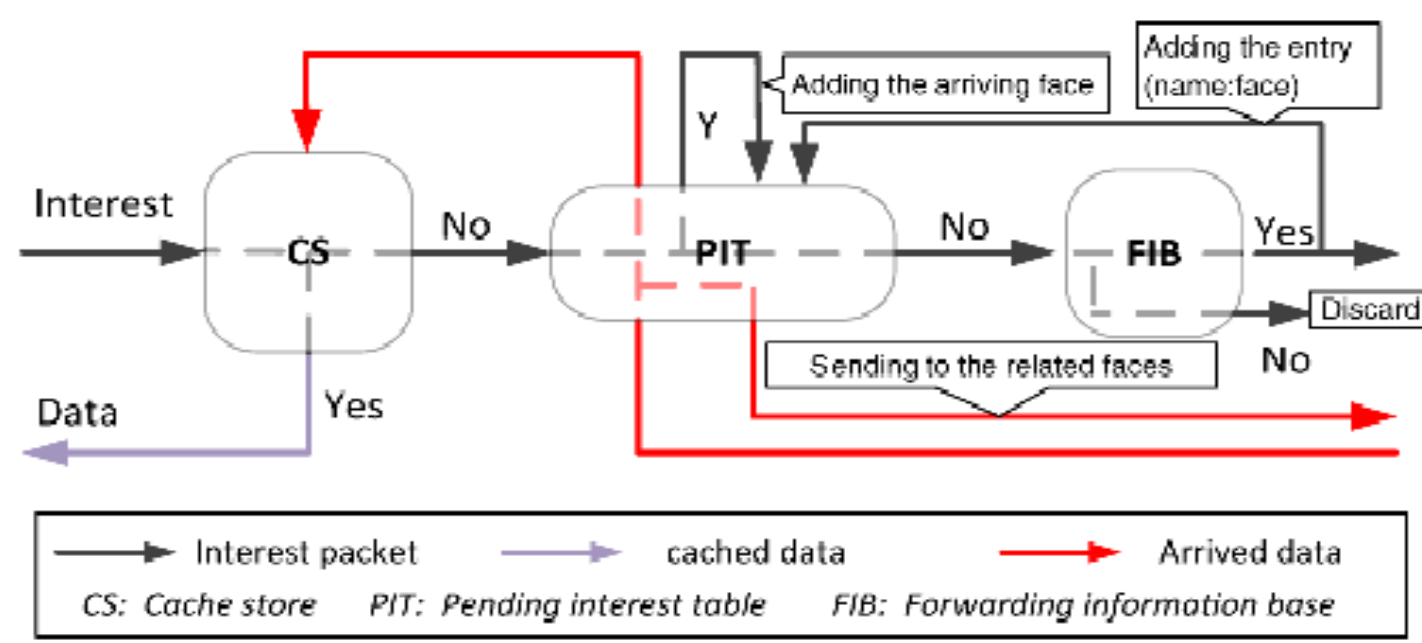

Figure 2: Packet Routing.

Every time consumer's interest packet is routed and its entry is checked in the FIB as a name prefix. The interest packet is routed or forwarded till it reaches the node that has exact content which consumer demands or that node generates the desired content for consumer. The forwarded data packet to the consumer is stored in the cache, that is done by the router for the future request made by other consumers. Thus the $\mathrm{NDN}$ is known for the technique called as name based content addressing technique. NDN is itself ad hoc and delay-tolerant related. That is why NDN is not centralize data server. It serves all the requests coming from the different consumers and cached all the content from anywhere.

\section{MQTT Protocol}

MQTT is a publish/subscribe messaging transport protocol based on Client Server architecture. MQTT is light weight protocol and is easy to implement. MQTT stands for Message Queuing Telemetry Transport. This protocol is designed in such a way to provide connectivity between physical devices with applications that are used in making web development. MQTT protocol is widely used in connectivity between IOT and M2M. Machine-to-Machine technology describes communication among machines. Vehicle-to-Vehicle communication is one of the example for M2M technology. It describes how one vehicle communicate with other vehicle using number of protocols (TCP/IP, HTTPs, MQTT, CoAP) available in network.In comparison with HTTPs, MQTT and CoAP are much better in communication. MQTT is having Client Server architecture where sensors act as clients and then connects to a server which is known as broker. sensor is a client and connects to a server, known as a broker.

MQTT is totally message oriented protocol where broker plays an important role. In vehicle-to-vehicle communication, MQTT also plays a vital role in IoT vehicular information network.

\subsection{Advantages}

- MQTT consumes minium battery than HTTPs.

- MQTT gives real time information.
- Messages are based on Store and Forward pattern.

- It delivers packets exactly to destination.

\section{Conclusion}

The high information management overhead and low communication efficiency incurred within the vehicular information network are challenging researcher, to investigate more efficient and scalable network architectures and communication schemes. To address some of the challenges, a Novel Framework for IoT vehicular information network based on MQTT protocol is proposed.

\section{References}

[1] Z. Yan, S. Zeadally, and Y. J. Park, "A Novel Vehicular Information Network Architecture Based on Named Data Networking (NDN)", IEEE internet of things journal, VOL. 1, NO. 6, Dec. 2014.

[2] L.Zhang, A Afanasyev, J Bruke, V Jacobson, “ VANETS via Named Data Networking”, ACM SIGCOMM VOL. 44, NO.3, July 2014.

[3] Lixia Zhang, Alexander Afanasyev, Jeffrey Burke, Van Jacobson,"Named Data Networking," ACM SIGCOMM Computer Communication Review, Volume 44, Number 3, July 2014.

[4] George Xylomenos, Christopher N. Ververidis, Vasilios A. Siris, Nikos Fotiou, Christos Tsilopoulos," A Survey of Information-Centric Networking Research" IEEE COMMUNICATIONS SURVEYS \& TUTORIALS, VOL. 16, NO. 2, SECOND QUARTER 2014

[5] G.Grassi*, Davide Pesavento*, Lucas Wang*, Giovanni Pau*," Vehicular Inter-Networking via Named Data," Comput.Sci.Dept, University of California, Los Angeles, CA, USA,2013.

[6] M. Meisel, V. Pappas, and L. Zhang, "Listen first, broadcast later: Topology-agnostic forwarding under high dynamics," Comput. Sci. Dept., UCLA, Los Angeles, CA, USA, Tech. Rep. 100021, 2010.

[7] L. Wang et al., "Rapid traffic information dissemination using named data," in Proc. 1st ACM NoM , Hilton Head, SC, USA, Jun. 11-14, 2012, pp. 7-12.

[8] S. Biswas, R . Tatchikou, and F. Dion, "Vehicle-tovehicle wireless communication protocols for enhancing 


\section{International Journal of Science and Research (IJSR) \\ ISSN (Online): 2319-7064}

Index Copernicus Value (2013): 6.14 | Impact Factor (2014): 5.611

highway traffic safety," IEEE Commun. Mag., vol. 44, no. 1, pp. 74-82, Jan. 2006.

[9] Teemu Koponen, Byung-Gon Chun, Andrey Ermolinskiy, " A Data-Oriented (and Beyond) Network Architecture," SIGCOMM'07, August 27-31, 2007, Kyoto, Japan.

[10] Katie Shilton, Jeff Burke, kc claffy," A World on NDN," Technical Report NDN-0018, Revision 1: 11 April 2014. 